\title{
Positive solutions for a system of nonlinear semipositone fractional q-difference equations with q-integral boundary conditions
}

\author{
Wei Cheng ${ }^{\mathrm{a}, *}$, Jiafa Xü ${ }^{\mathrm{a}}$ Yujun Cui ${ }^{\mathrm{b}, \mathrm{c}}$ \\ a School of Mathematical Sciences, Chongqing Normal University, Chongqing 401331, China. \\ ${ }^{b}$ State Key Laboratory of Mining Disaster Prevention and Control Co-founded by Shandong Province, China. \\ ${ }^{c}$ Ministry of Science and Technology, Shandong University of Science and Technology, Qingdao 266590, China. \\ Communicated by J. Brzdek
}

\begin{abstract}
In this paper, by virtue of fixed point index on cones, we obtain two existence theorems of positive solutions for a system of nonlinear semipositone fractional q-difference equations with q-integral boundary conditions. Concave functions and nonnegative matrices are used to characterize the coupling behavior of our nonlinearities. (c)2017 All rights reserved.
\end{abstract}

Keywords: q-difference equation, q-integral boundary conditions, fixed point index, positive solution, concave function. 2010 MSC: 34B10, 34B18, 34A34, 45G15, 45M20.

\section{Introduction}

In this work we study the following system of nonlinear semipositone fractional q-difference equations with q-integral boundary conditions

$$
\left\{\begin{array}{l}
D_{q}^{\alpha} u(t)+f_{1}(t, u(t), v(t))=0, t \in(0,1) \\
D_{q}^{\alpha} v(t)+f_{2}(t, u(t), v(t))=0, t \in(0,1) \\
u(0)=0, D_{q} u(0)=0, D_{q}^{v} u(1)=\beta \int_{0}^{1} D_{q}^{v} u(t) d_{q} t \\
v(0)=0, D_{q} v(0)=0, D_{q}^{v} v(1)=\beta \int_{0}^{1} D_{q}^{v} v(t) d_{q} t
\end{array}\right.
$$

where $\alpha \in(2,3), v \in(1,2)$ are real numbers, $D_{q}^{\alpha}$ is the Riemann-Liouville's fractional $q$-derivative of order $\alpha$, and the nonnegative constant $\beta$, and the functions $f_{i}(i=1,2)$ satisfy the conditions

(H1) $\beta \geqslant 0$ and $1-\beta \int_{0}^{1} t^{\alpha-\nu-1} d_{q} t:=A>0$;

\footnotetext{
*Corresponding author

Email addresses: 1375415619@qq.com (Wei Cheng), xujiafa292@sina.com (Jiafa Xu), cyj720201@163.com (Yujun Cui) doi:10.22436/jnsa.010.08.35
} 
(H2) $f_{i} \in C\left([0,1] \times \mathbb{R}^{+} \times \mathbb{R}^{+}, \mathbb{R}\right)$ and there exists $M>0$ such that

$$
f_{i}(t, x, y) \geqslant-M, \forall(t, x, y) \in[0,1] \times \mathbb{R}^{+} \times \mathbb{R}^{+}, i=1,2 .
$$

Recently, there are a large number of papers involving fractional differential equations in the literatures, for example, we refer the readers to [1-5, 7-25] and the references therein. In [7, 14], Henderson and Luca had studied the system of fractional differential equations with sign-changing nonlinearities

$$
\left\{\begin{array}{l}
D_{0+}^{\alpha} u(t)+\lambda f(t, u(t), v(t))=0, t \in(0,1) \\
D_{0+}^{\beta} v(t)+\mu g(t, u(t), v(t))=0, t \in(0,1) \\
u(0)=u^{(i)}(0)=0, u^{\prime}(1)=\int_{0}^{1} v(s) d H(s), i=1,2, \ldots, n-2, \\
v(0)=v^{(i)}(0)=0, v^{\prime}(1)=\int_{0}^{1} u(s) d K(s), \quad i=1,2, \ldots, m-2
\end{array}\right.
$$

and they obtained the existence of positive solutions by the nonlinear alternative of Leray-Schauder type and the Guo-Krasnosel'skii fixed point theorem. Their nonlinearities $f, g$ are superlinear growth depending on the unknown functions $u, v$, i.e.,

$$
f_{\infty}=\lim _{u+v \rightarrow \infty} \min _{t \in[c, 1-c]} \frac{f(t, u, v)}{u+v}=\infty, g_{\infty}=\lim _{u+v \rightarrow \infty} \min _{t \in[c, 1-c]} \frac{g(t, u, v)}{u+v}=\infty, c \in\left(0, \frac{1}{2}\right) .
$$

In $[3,19-21]$, the authors had adopted the similar conditions of (1.2) to obtain various existence theorems of positive solutions for some semipositone fractional boundary value problems.

In [25], Zhang et al. had studied the system of fractional differential equations with Riemann-Liouville fractional derivative

$$
\left\{\begin{array}{l}
D_{0+}^{\alpha} D_{0+}^{\alpha} x(t)=f\left(t, x, x^{\prime},-D_{0+}^{\alpha} x, y, y^{\prime},-D_{0+}^{\alpha} y\right)=0, t \in(0,1) \\
D_{0+}^{\alpha} D_{0+}^{\alpha} y(t)=g\left(t, x, x^{\prime},-D_{0+}^{\alpha} x, y, y^{\prime},-D_{0+}^{\alpha} y\right)=0, t \in(0,1) \\
x(0)=x^{\prime}(0)=x^{\prime}(1)=D_{0+}^{\alpha} x(0)=D_{0+}^{\alpha+1} x(0)=D_{0+}^{\alpha+1} x(1)=0 \\
y(0)=y^{\prime}(0)=y^{\prime}(1)=D_{0+}^{\alpha} y(0)=D_{0+}^{\alpha+1} y(0)=D_{0+}^{\alpha+1} y(1)=0 .
\end{array}\right.
$$

They used the Krasnoselskii-Zabreiko fixed point theorem to establish some existence theorems of positive solutions for (1.3).

In this paper, inspired by the above works, we investigate the existence of positive solutions for (1.1) by fixed point index on cones. Moreover, some appropriate concave functions and nonnegative matrices are used to depict the coupling behavior of our nonlinearities, and then our nonlinearities grow both superlinearly and sublinearly.

\section{Preliminaries}

Let $\mathrm{q} \in(0,1)$ and define

$$
[a]_{q}=\frac{1-q^{a}}{1-q}, \quad a \in \mathbb{R} .
$$

The q-analogue of the power function $(a-b)^{n}$ with $\mathbb{N}_{0}$ is

$$
(a-b)^{0}=1, \quad(a-b)^{n}=\prod_{k=0}^{n-1}\left(a-b q^{k}\right), \quad n \in \mathbb{N}, a, b \in \mathbb{R} .
$$

More generally, if $\alpha \in \mathbb{R}$, then

$$
(a-b)^{(\alpha)}=a^{\alpha} \prod_{n=0}^{\infty} \frac{a-b q^{n}}{a-b q^{\alpha+n}}
$$


Note that, if $b=0$ then $a^{(\alpha)}=a^{\alpha}$. The q-gamma function is defined by

$$
\Gamma_{\mathrm{q}}(\mathrm{x})=\frac{(1-\mathrm{q})^{(\mathrm{x}-1)}}{(1-\mathrm{q})^{\mathrm{x}-1}}, \quad x \in \mathbb{R} \backslash\{0,-1,-2, \ldots\},
$$

and satisfies $\Gamma_{\mathrm{q}}(\mathrm{x}+1)=[\mathrm{x}] \Gamma_{\mathrm{q}}(\mathrm{x})$. The $\mathrm{q}$-derivative of a function $\mathrm{f}$ is here defined by

$$
\left(D_{q} f\right)(x)=\frac{f(x)-f(q x)}{(1-q) x}, \quad\left(D_{q} f\right)(0)=\lim _{x \rightarrow 0}\left(D_{q} f\right)(x)
$$

and q-derivatives of higher order by

$$
\left(D_{q}^{0} f\right)(x)=f(x) \quad \text { and } \quad\left(D_{q}^{n} f\right)(x)=D_{q}\left(D_{q}^{n-1} f\right)(x), \quad n \in \mathbb{N} .
$$

The $q$-integral of a function $f$ defined in the interval $[0, b]$ is given by

$$
\left(I_{q} f\right)(x)=\int_{0}^{x} f(t) d_{q} t=x(1-q) \sum_{n=0}^{\infty} f\left(x q^{n}\right) q^{n}, \quad x \in[0, b] .
$$

If $a \in[0, b]$ and $f$ is defined in the interval $[0, b]$, its integral from $a$ to $b$ is defined by

$$
\int_{a}^{b} f(t) d_{q} t=\int_{0}^{b} f(t) d_{q} t-\int_{0}^{a} f(t) d_{q} t
$$

Similarly as done for derivatives, an operator $\mathrm{I}_{\mathrm{q}}^{\mathrm{n}}$ can be defined, namely,

$$
\left(I_{q}^{0} f\right)(x)=f(x) \quad \text { and }\left(I_{q}^{n} f\right)(x)=I_{q}\left(I_{q}^{n-1} f\right)(x), \quad n \in \mathbb{N} .
$$

The fundamental theorem of calculus is applied to these operators $I_{q}$ and $D_{q}$; i.e.,

$$
\left(D_{q} I_{q} f\right)(x)=f(x),
$$

and if $f$ is continuous at $x=0$, then

$$
\left(I_{q} D_{q} f\right)(x)=f(x)-f(0)
$$

Basic properties of the two operators can be found in the book [9]. We now point out three formulas that will be used later $\left({ }_{i} D_{q}\right.$ denotes the derivative with respect to variable $i$ )

$$
\begin{aligned}
{[a(t-s)]^{(\alpha)} } & =a^{\alpha}(t-s)^{(\alpha)}, \\
{ }_{t} D_{q}(t-s)^{(\alpha)} & =[\alpha]_{q}(t-s)^{(\alpha-1)}, \\
\left({ }_{x} D_{q} \int_{0}^{x} f(x, t) d_{q} t\right)(x) & =\int_{0}^{x}{ }_{x} D_{q} f(x, t) d_{q} t+f(q x, x) .
\end{aligned}
$$

Remark 2.1 ([4]). We note that if $\alpha>0$ and $a \leqslant b \leqslant t$, then $(t-a)^{(\alpha)} \geqslant(t-b)^{(\alpha)}$.

Definition 2.2 ([1]). Let $\alpha \geqslant 0$ and $f$ be a function defined on $[0,1]$. The fractional $q$-integral of the Riemann-Liouville type is $\left(\mathrm{I}_{\mathrm{q}}^{0} \mathrm{f}\right)(\mathrm{x})=\mathrm{f}(\mathrm{x})$ and

$$
\left(I_{q}^{\alpha} f\right)(x)=\frac{1}{\Gamma_{q}(\alpha)} \int_{0}^{x}(x-q t)^{(\alpha-1)} f(t) d_{q} t, \quad \alpha>0, x \in[0,1] .
$$

Definition 2.3 ([16]). The fractional q-derivative of the Riemann-Liouville type of order $\alpha \geqslant 0$ is defined by $\left(D_{q}^{0} f\right)(x)=f(x)$ and

$$
\left(D_{q}^{\alpha} f\right)(x)=\left(D_{q}^{m} I_{q}^{m-\alpha} f\right)(x), \quad \alpha>0,
$$

where $m$ is the smallest integer greater than or equal to $\alpha$. 
Next, we list some properties that are already known in the literature, for more details, please refer to $[1,4,16]$.

Lemma 2.4. Let $\alpha, \beta \geqslant 0$ and $\mathrm{f}$ be a function defined on $[0,1]$. Then the following formulas hold:

(1) $\left(I_{q}^{\beta} I_{q}^{\alpha} f\right)(x)=\left(I_{q}^{\alpha+\beta} f\right)(x)$,

(2) $\left(D_{q}^{\alpha} I_{q}^{\alpha} f\right)(x)=f(x)$.

Lemma 2.5. Let $\alpha>0$ and $\mathrm{p}$ be a positive integer. Then the following equality holds:

$$
\left(I_{q}^{\alpha} D_{q}^{p_{f}} f\right)(x)=\left(D_{q}^{p} I_{q}^{\alpha} f\right)(x)-\sum_{k=0}^{p-1} \frac{x^{\alpha-p+k}}{\Gamma_{q}(\alpha+k-p+1)}\left(D_{q}^{k} f\right)(0) .
$$

Next, we study the following fractional boundary value problems

$$
\left\{\begin{array}{l}
D_{q}^{\alpha} u(t)+f(t, u(t))=0, t \in(0,1), \\
u(0)=0, D_{q} u(0)=0, D_{q}^{v} u(1)=\beta \int_{0}^{1} D_{q}^{v} u(t) d_{q} t,
\end{array}\right.
$$

where $\beta$, f satisfy $(\mathrm{H} 1)$ and

$(\mathrm{H} 2)^{\prime} \mathrm{f} \in \mathrm{C}\left([0,1] \times \mathbb{R}^{+}, \mathbb{R}\right)$ and there exists $M>0$ such that

$$
f(t, x) \geqslant-M, \forall(t, x) \in[0,1] \times \mathbb{R}^{+} .
$$

Lemma 2.6. Let $\alpha \in(2,3), v \in(1,2)$. Then (2.1) is equivalent to the Hammerstein integral equation

$$
u(t)=\int_{0}^{1} G(t, q s) f(s, u(s)) d_{q} s, \forall t \in[0,1]
$$

where

$$
\begin{aligned}
& G(t, s)=H_{1}(t, s)+\frac{\beta t^{\alpha-1}}{A}\left[\int_{0}^{1} H_{2}(t, s) d_{q} t\right], \\
& H_{1}(t, s)=\frac{1}{\Gamma_{q}(\alpha)} \begin{cases}t^{\alpha-1}(1-s)^{(\alpha-v-1)}-(t-s)^{(\alpha-1)}, & 0 \leqslant s \leqslant t \leqslant 1, \\
t^{\alpha-1}(1-s)^{(\alpha-v-1)}, & 0 \leqslant t \leqslant s \leqslant 1,\end{cases} \\
& H_{2}(t, s)=\frac{1}{\Gamma_{q}(\alpha)} \begin{cases}t^{\alpha-v-1}(1-s)^{(\alpha-v-1)}-(t-s)^{(\alpha-v-1)}, & 0 \leqslant s \leqslant t \leqslant 1, \\
t^{\alpha-v-1}(1-s)^{(\alpha-v-1)}, & 0 \leqslant t \leqslant s \leqslant 1 .\end{cases}
\end{aligned}
$$

Proof. From the definitions and properties of the fractional q-derivative of the Riemann-Liouville type, we have

$$
u(t)=c_{1} t^{\alpha-1}+c_{2} t^{\alpha-2}+c_{3} t^{\alpha-3}-I_{q}^{\alpha} f(t, u(t)), c_{i} \in \mathbb{R}, i=1,2,3 .
$$

The conditions $u(0)=0, D_{q} u(0)=0$ enable us to obtain $c_{2}=c_{3}=0$. Hence,

$$
u(t)=c_{1} t^{\alpha-1}-I_{q}^{\alpha} f(t, u(t)),
$$

and

$$
D_{q}^{v} u(t)=\frac{\Gamma_{q}(\alpha)}{\Gamma_{q}(\alpha-v)} c_{1} t^{\alpha-v-1}-\frac{1}{\Gamma_{q}(\alpha-v)} \int_{0}^{t}(t-q s)^{(\alpha-v-1)} f(s, u(s)) d_{q} s .
$$

From $D_{q}^{v} u(1)=\beta \int_{0}^{1} D_{q}^{v} u(t) d_{q} t$ we have

$$
\begin{aligned}
& \frac{\Gamma_{\mathrm{q}}(\alpha)}{\Gamma_{\mathrm{q}}(\alpha-v)} \mathrm{c}_{1}-\frac{1}{\Gamma_{\mathrm{q}}(\alpha-v)} \int_{0}^{1}(1-\mathrm{qs})^{(\alpha-v-1)} \mathrm{f}(\mathrm{s}, \mathrm{u}(\mathrm{s})) \mathrm{d}_{\mathrm{q}} \mathrm{s} \\
& \quad=\beta \frac{\Gamma_{\mathrm{q}}(\alpha)}{\Gamma_{\mathrm{q}}(\alpha-v)} \mathrm{c}_{1} \int_{0}^{1} \mathrm{t}^{\alpha-v-1} \mathrm{~d}_{\mathrm{q}} \mathrm{t}-\frac{\beta}{\Gamma_{\mathrm{q}}(\alpha-v)} \int_{0}^{1} \int_{0}^{\mathrm{t}}(\mathrm{t}-\mathrm{qs})^{(\alpha-v-1)} \mathrm{f}(\mathrm{s}, \mathrm{u}(\mathrm{s})) \mathrm{d}_{\mathrm{q}} s \mathrm{~d}_{\mathrm{q}} \mathrm{t},
\end{aligned}
$$

and 


$$
\begin{aligned}
c_{1} & =\frac{1}{A \Gamma_{q}(\alpha)} \int_{0}^{1}(1-q s)^{(\alpha-v-1)} f(s, u(s)) d_{q} s-\frac{\beta}{A \Gamma_{q}(\alpha)} \int_{0}^{1} \int_{0}^{t}(t-q s)^{(\alpha-v-1)} f(s, u(s)) d_{q} s d_{q} t \\
& =\frac{1}{A \Gamma_{q}(\alpha)} \int_{0}^{1}(1-q s)^{(\alpha-v-1)} f(s, u(s)) d_{q} s-\frac{\beta}{A \Gamma_{q}(\alpha)} \int_{0}^{1} \int_{s}^{1}(t-q s)^{(\alpha-v-1)} f(s, u(s)) d_{q} t d_{q} s .
\end{aligned}
$$

Therefore,

$$
\begin{aligned}
u(t)= & \frac{1}{A \Gamma_{q}(\alpha)} \int_{0}^{1} t^{\alpha-1}(1-q s)^{(\alpha-v-1)} f(s, u(s)) d_{q} s-\frac{\beta t^{\alpha-1}}{A \Gamma_{q}(\alpha)} \int_{0}^{1} \int_{s}^{1}(t-q s)^{(\alpha-v-1)} f(s, u(s)) d_{q} t d_{q} s \\
& -\frac{1}{\Gamma_{q}(\alpha)} \int_{0}^{t}(t-q s)^{(\alpha-1)} f(s, u(s)) d_{q} s+\frac{1}{\Gamma_{q}(\alpha)} \int_{0}^{1} t^{\alpha-1}(1-q s)^{(\alpha-v-1)} f(s, u(s)) d_{q} s \\
& -\frac{1}{\Gamma_{q}(\alpha)} \int_{0}^{1} t^{\alpha-1}(1-q s)^{(\alpha-v-1)} f(s, u(s)) d_{q} s \\
= & \int_{0}^{1} H_{1}(t, q s) f(s, u(s)) d_{q} s \\
& +\frac{\beta t^{\alpha-1}}{A \Gamma_{q}(\alpha)} \int_{0}^{1}\left[\int_{0}^{1} t^{\alpha-v-1}(1-q s)^{(\alpha-v-1)} d_{q} t-\int_{s}^{1}(t-q s)^{(\alpha-v-1)} d_{q} t\right] f(s, u(s)) d_{q} s \\
= & \int_{0}^{1} H_{1}(t, q s) f(s, u(s)) d_{q} s+\int_{0}^{1} \frac{\beta t^{\alpha-1}}{A}\left[\int_{0}^{1} H_{2}(t, q s) d_{q} t\right] f(s, u(s)) d_{q} s \\
= & \int_{0}^{1} G(t, q s) f(s, u(s)) d_{q} s .
\end{aligned}
$$

Lemma 2.7 ([5]). The functions $\mathrm{H}_{i}(t, s)(i=1,2)$ in (2.3)-(2.4) satisfy the following properties:

(1) $\mathrm{H}_{\mathrm{i}}(\mathrm{t}, \mathrm{qs}) \geqslant 0$ for $\mathrm{t}, \mathrm{s} \in[0,1]$;

(2) $\mathrm{t}^{\alpha-1} \mathrm{H}_{1}(1, \mathrm{qs}) \leqslant \mathrm{H}_{1}(\mathrm{t}, \mathrm{qs}) \leqslant \mathrm{H}_{1}(1, \mathrm{qs})$ for $\mathrm{t}, \mathrm{s} \in[0,1]$;

(3) $\mathrm{H}_{1}(\mathrm{t}, \mathrm{qs}) \leqslant \frac{1}{\Gamma_{\mathrm{q}}(\alpha)} \mathrm{t}^{\alpha-1}(1-\mathrm{s})^{(\alpha-v-1)}$ for $\mathrm{t}, \mathrm{s} \in[0,1]$.

Proof. The proof is analogous to the proof of Lemma 3.0.7 in [5], and hence, we omitted it.

From Lemma 2.7, for all $t, s \in[0,1]$, we have

$$
t^{\alpha-1}\left(H_{1}(1, q s)+\frac{\beta}{A}\left[\int_{0}^{1} H_{2}(t, q s) d_{q} t\right]\right) \leqslant G(t, q s) \leqslant H_{1}(1, q s)+\frac{\beta}{A}\left[\int_{0}^{1} H_{2}(t, q s) d_{q} t\right],
$$

and

$$
G(t, q s) \leqslant t^{\alpha-1}\left(\frac{1}{\Gamma_{q}(\alpha)}(1-s)^{(\alpha-v-1)}+\frac{\beta}{A}\left[\int_{0}^{1} H_{2}(t, q s) d_{q} t\right]\right) .
$$

For convenience, we let

$$
\varphi(s)=H_{1}(1, q s)+\frac{\beta}{A}\left[\int_{0}^{1} H_{2}(t, q s) d_{q} t\right], \phi(s)=\frac{1}{\Gamma_{q}(\alpha)}(1-s)^{(\alpha-v-1)}+\frac{\beta}{A}\left[\int_{0}^{1} H_{2}(t, q s) d_{q} t\right], \forall s \in[0,1] .
$$

Subsequently, we have

$$
\kappa_{1} \varphi(s) \leqslant \int_{0}^{1} G(t, q s) \varphi(t) d_{q} t \leqslant \kappa_{2} \varphi(s) \text { for } s \in[0,1]
$$


where $\kappa_{1}=\int_{0}^{1} t^{\alpha-1} \varphi(t) d_{q} t, k_{2}=\int_{0}^{1} \varphi(t) d_{q} t$.

Let $E:=C[0,1],\|u\|:=\max _{t \in[0,1]}|u(t)|$ and $P=\{u \in E: u(t) \geqslant 0, \forall t \in[0,1]\}$. Then $(E,\|\cdot\|)$ is a real Banach space and $\mathrm{P}$ is a cone in $\mathrm{E}$.

Let $F(t, z)=\left\{\begin{array}{l}f(t, z)+M, z \geqslant 0, \\ f(t, 0)+M, z \leqslant 0,\end{array}\right.$ and $w(t)=M \int_{0}^{1} G(t, q s) d_{q}$ s for $t \in[0,1]$. Then we consider the boundary value problem

$$
\left\{\begin{array}{l}
D_{q}^{\alpha} u(t)+F(t, u(t)-w(t))=0, t \in(0,1) \\
u(0)=0, D_{q} u(0)=0, D_{q}^{v} u(1)=\beta \int_{0}^{1} D_{q}^{v} u(t) d_{q} t
\end{array}\right.
$$

where $\beta$ satisfies (H1). From Lemma 2.6, (2.7) is equivalent to the Hammerstein integral equation

$$
u(t)=\int_{0}^{1} G(t, q s) F(s, u(s)-w(s)) d_{q} s:=(\mathcal{B} u)(t) \text { for } t \in[0,1] .
$$

Then $\mathcal{B}: \mathrm{P} \rightarrow \mathrm{P}$ is a completely continuous operator. Moreover the following claims are true:

(1) if $u^{*}$ is a positive solution of (2.1), then $u^{*}+w$ is a positive fixed point of $\mathcal{B}$;

(2) if $u$ is a positive fixed point of $\mathcal{B}$ and $u(t) \geqslant w(t), t \in[0,1]$, then $u^{*}=u-w$ is a positive solution of (2.1).

Lemma 2.8. Let $\mathrm{P}_{0}:=\left\{\mathrm{u} \in \mathrm{P}: \mathrm{u}(\mathrm{t}) \geqslant \mathrm{t}^{\alpha-1}\|\mathrm{u}\|\right\}$. Then $\mathrm{P}_{0}$ is a cone in $\mathrm{E}$ and $\mathcal{B}(\mathrm{u}) \subset \mathrm{P}_{0}$.

To obtain a positive solution of (2.1), we seek a positive fixed point $u$ of $\mathcal{B}$ with $u \geqslant w$. From (2.5), for any $u \in \mathrm{P}_{0}$ we have

$$
\begin{aligned}
u(t)-w(t) & =u(t)-M \int_{0}^{1} G(t, q s) d_{q} s \\
& \geqslant u(t)-M \int_{0}^{1} t^{\alpha-1} \phi(s) d_{q} s=u(t)-M t^{\alpha-1} \int_{0}^{1} \phi(s) d_{q} s \geqslant u(t)-M \int_{0}^{1} \phi(s) d_{q} s \frac{u(t)}{\|u\|} .
\end{aligned}
$$

As a result, $u(t) \geqslant w(t)$ for $t \in[0,1]$ if $\|u\| \geqslant M \int_{0}^{1} \phi(s) d_{q} s:=\kappa_{3}$.

Lemma 2.9 ([6]). Let $\Omega \subset \mathrm{E}$ be a bounded open set and $\mathrm{A}: \bar{\Omega} \cap \mathrm{P} \rightarrow \mathrm{P}$ a completely continuous operator. If there exists $u_{0} \in P \backslash\{0\}$ such that $u-A u \neq \mu u_{0}$ for all $\mu \geqslant 0$ and $u \in \partial \Omega \cap P$, then $i(A, \Omega \cap P, P)=0$, where $i$ denotes the fixed point index on $\mathrm{P}$.

Lemma 2.10 ([6]). Let $\Omega \subset \mathrm{E}$ be a bounded open set with $0 \in \Omega$. Suppose $\mathrm{A}: \bar{\Omega} \cap \mathrm{P} \rightarrow \mathrm{P}$ is a completely continuous operator. If $u \neq \mu A u$ for all $u \in \partial \Omega \cap P$ and $0 \leqslant \mu \leqslant 1$, then $i(A, \Omega \cap P, P)=1$.

\section{Main results}

From Lemma 2.6, we see that (1.1) is equivalent to the system of Hammerstein integral equations

$$
\left\{\begin{array}{l}
u(t)=\int_{0}^{1} G(t, q s) f_{1}(s, u(s), v(s)) d_{q} s \\
v(t)=\int_{0}^{1} G(t, q s) f_{2}(s, u(s), v(s)) d_{q} s
\end{array}\right.
$$

where $G$ is defined by (2.2). Let $F_{i}(t, x, y)=\left\{\begin{array}{l}f_{i}(t, x, y)+M, x, y \geqslant 0, \\ f_{i}(t, 0,0)+M, x, y \leqslant 0 .\end{array}\right.$ Then we can define the operators

$$
\mathcal{T}_{\mathfrak{i}}(u, v)(t)=\int_{0}^{1} \mathrm{G}(\mathrm{t}, \mathrm{qs}) \mathrm{F}_{\mathrm{i}}(\mathrm{s}, \mathrm{u}(\mathrm{s})-w(\mathrm{~s}), v(\mathrm{~s})-w(s)) \mathrm{d}_{\mathrm{q}} \mathrm{s} \text { for } u, v \in \mathrm{E}
$$


and

$$
\mathcal{T}(u, v)(t)=\left(\mathcal{T}_{1}, \mathcal{T}_{2}\right)(u, v)(t) \text { for } u, v \in E
$$

Therefore, if $(u, v)$ is a positive fixed point for $\mathcal{T}$ with $u(t) \geqslant w(t), v(t) \geqslant w(t)$ for $t \in[0,1]$, then $\left(u^{*}, v^{*}\right)=$ $(u-w, v-w)$ is a positive solution for (1.1). Moreover, from the continuity of $G$ and $F_{i}(i=1,2)$, we know that $\mathcal{T}_{i}: \mathrm{P} \times \mathrm{P} \rightarrow \mathrm{P}, \mathcal{T}: \mathrm{P} \times \mathrm{P} \rightarrow \mathrm{P} \times \mathrm{P}$ are completely continuous operators.

Let $\mathcal{K}:=\max _{\mathrm{t}, \mathrm{s} \in[0,1]} \mathrm{G}(\mathrm{t}, \mathrm{qs}), \mathrm{B}_{\rho}:=\{v \in \mathrm{E}:\|v\|<\rho\}$ for $\rho>0$. In the sequel, we use $\mathrm{c}_{1}, \mathrm{c}_{1}^{\prime}, \mathrm{c}_{2}, \ldots, \mathrm{d}_{1}$, $d_{2}, \ldots$ to stand for different positive constants. Now, we list our assumptions on $F_{i}(i=1,2)$.

(H3) There exist $p, q \in C\left(\mathbb{R}^{+}, \mathbb{R}^{+}\right)$such that

(i) $p$ is concave and strictly increasing on $\mathbb{R}^{+}$;

(ii) there exist $d_{1}>0, d_{2}>0, c_{1}>0$ such that $d_{1} d_{2} \kappa_{1}^{2}>1$ and

$$
F_{1}(t, x, y) \geqslant d_{1} p(y)-c_{1}, F_{2}(t, x, y) \geqslant d_{2} q(x)-c_{1}, \forall(t, x, y) \in[0,1] \times \mathbb{R}^{+} \times \mathbb{R}^{+} ;
$$

(iii) $p\left(d_{2} \mathcal{K} q(u(s)-w(s))\right) \geqslant d_{2} \mathcal{K}(u(s)-w(s))-c_{1}$ for $u \in \mathbb{R}^{+}$.

(H4) There exists $\mathcal{M}_{1} \in\left(0, \kappa_{3} \kappa_{2}^{-1}\right)$ such that

$$
F_{i}(t, x, y) \leqslant \mathcal{M}_{1}, \forall(t, x, y) \in[0,1] \times\left[0, k_{3}\right] \times\left[0, k_{3}\right], i=1,2 .
$$

(H5) There exist $\mathcal{M}_{2}>0, \theta \in(0,1)$ and $t_{0} \in[\theta, 1]$ such that $t_{0}^{\alpha-1} \mathcal{M}_{2} \int_{\theta}^{1} \varphi(s) d_{q} s \geqslant k_{3}$ and

$$
F_{i}(t, x, y) \geqslant \mathcal{M}_{2} \text { for }(t, x, y) \in[\theta, 1] \times\left[0, k_{3}\right] \times\left[0, \kappa_{3}\right], i=1,2 .
$$

(H6) There exist $d_{i}>0(i=3,4,5,6)$ and $c_{1}^{\prime}>0$ such that

$$
\kappa_{2} d_{3}<1, \kappa_{2} d_{6}<1, \Delta:=\left|\begin{array}{cc}
1-\kappa_{2} d_{3} & -\kappa_{2} d_{4} \\
-\kappa_{2} d_{5} & 1-\kappa_{2} d_{6}
\end{array}\right|>0,
$$

and

$$
F_{1}(t, x, y) \leqslant d_{3} x+d_{4} y+c_{1}^{\prime}, F_{2}(t, x, y) \leqslant d_{5} x+d_{6} y+c_{1}^{\prime}, \forall(t, x, y) \in[0,1] \times \mathbb{R}^{+} \times \mathbb{R}^{+} .
$$

Theorem 3.1. Suppose that (H1)-(H4) hold. Then (1.1) has at least one positive solution.

Proof. We first want to show that there exists an adequately big positive number $\mathrm{R}>\mathrm{K}_{3}$ such that

$$
(u, v) \neq \mathcal{T}(u, v)+\lambda(\psi, \psi), \forall(u, v) \in \partial B_{R} \cap(P \times P), \lambda \geqslant 0,
$$

where $\psi \in P_{0}$ is a given function. Indeed, if not, there exist $(u, v) \in \partial B_{R} \cap(P \times P)$ and $\lambda \geqslant 0$ such that $(u, v)=\mathcal{T}(u, v)+\lambda(\psi, \psi)$, and then $u(t) \geqslant \mathcal{T}_{1}(u, v)(t)$, and $v(t) \geqslant \mathcal{T}_{2}(u, v)(t)$ for $t \in[0,1]$. In view of $(H 3)$, we have

$$
\begin{aligned}
u(t) & \geqslant \int_{0}^{1} G(t, q s)\left(d_{1} p(v(s)-w(s))-c_{1}\right) d_{q} s \\
& \geqslant \int_{0}^{1} G(t, q s)\left(d_{1}[p(v(s))-p(w(s))]-c_{1}\right) d_{q} s \geqslant d_{1} \int_{0}^{1} G(t, q s) p(v(s)) d_{q} s-c_{2},
\end{aligned}
$$

and

$$
v(t) \geqslant \int_{0}^{1} G(t, q s)\left(d_{2} q(u(s)-w(s))-c_{1}\right) d_{q} s \geqslant d_{2} \int_{0}^{1} G(t, q s) q(u(s)-w(s)) d_{q} s-c_{3} .
$$

By the concavity of $p$, we obtain

$$
p\left(v(t)+c_{3}\right) \geqslant p\left(d_{2} \int_{0}^{1} G(t, q s) q(u(s)-w(s)) d_{q} s\right)
$$




$$
\begin{aligned}
& \geqslant \int_{0}^{1} p\left(d_{2} G(t, q s) q(u(s)-w(s))\right) d_{q} s \\
& =\int_{0}^{1} p\left(d_{2} \mathcal{K} \frac{G(t, q s)}{\mathcal{K}} q(u(s)-w(s))\right) d_{q} s \geqslant \int_{0}^{1} \frac{G(t, q s)}{\mathcal{K}} p\left(d_{2} \mathcal{K} q(u(s)-w(s))\right) d_{q} s .
\end{aligned}
$$

From (H3) (iii) we have

$$
\begin{aligned}
p(v(t)) & \geqslant p\left(v(t)+c_{3}\right)-p\left(c_{3}\right) \\
& \geqslant \int_{0}^{1} \frac{G(t, q s)}{\mathcal{K}} p\left(d_{2} \mathcal{K} q(u(s)-w(s))\right) d_{q} s-p\left(c_{3}\right) \\
& \geqslant \int_{0}^{1} \frac{G(t, q s)}{\mathcal{K}}\left[d_{2} \mathcal{K}(u(s)-w(s))-c_{1}\right] d_{q} s-p\left(c_{3}\right) \geqslant d_{2} \int_{0}^{1} G(t, q s)(u(s)-w(s)) d_{q} s-c_{4} .
\end{aligned}
$$

Combining this with (3.2), we obtain

$$
\begin{aligned}
u(t) & \geqslant d_{1} \int_{0}^{1} G(t, q s)\left(d_{2} \int_{0}^{1} G(s, q \tau)(u(\tau)-w(\tau)) d_{q} \tau-c_{4}\right) d_{q} s-c_{2} \\
& \geqslant d_{1} d_{2} \int_{0}^{1} G(t, q s) \int_{0}^{1} G(s, q \tau) u(\tau) d_{q} \tau d_{q} s-c_{5} .
\end{aligned}
$$

Now multiplying by $\varphi(t)$ and integrating over $[0,1]$, from $(2.6)$ we obtain

$$
\int_{0}^{1} \varphi(t) u(t) d_{q} t \geqslant d_{1} d_{2} \kappa_{1}^{2} \int_{0}^{1} \varphi(t) u(t) d_{q} t-c_{5} \kappa_{2}
$$

and thus

$$
\int_{0}^{1} \varphi(t) u(t) d_{q} t \leqslant \frac{c_{5} \kappa_{2}}{d_{1} d_{2} \kappa_{1}^{2}-1}
$$

Note that $u \in P_{0}$, we have

$$
\int_{0}^{1} \varphi(t) t^{\alpha-1}\|u\| d_{q} t \leqslant \int_{0}^{1} \varphi(t) u(t) d_{q} t \leqslant \frac{c_{5} \kappa_{2}}{d_{1} d_{2} \kappa_{1}^{2}-1},
$$

and

$$
\|u\| \leqslant \frac{c_{5} \kappa_{2}}{\kappa_{1}\left(d_{1} d_{2} \kappa_{1}^{2}-1\right)}
$$

On the other hand, by (3.2) we have

$$
\int_{0}^{1} G(t, q s) p(v(s)) d_{q} s \leqslant \frac{c_{2}+\|u\|}{d_{1}} \leqslant \frac{c_{2}+\frac{c_{5} \kappa_{2}}{k_{1}\left(d_{1} d_{2} \kappa_{1}^{2}-1\right)}}{d_{1}}:=\mathcal{N}_{1}, \forall t \in[0,1] .
$$

This implies

$$
\int_{0}^{1} \varphi(t) p(v(t)) d_{q} t \leqslant \mathcal{N}_{1}
$$

Note that we may assume $v(t) \not \equiv 0$ for $t \in[0,1]$. Then $\|v\|>0$ and $p(\|v\|)>0$. For $v \in \mathrm{P}_{0}$, we have

$$
\mathrm{k}_{1}\|v\| \leqslant \int_{0}^{1} \varphi(\mathrm{t}) v(\mathrm{t}) \mathrm{d}_{\mathrm{q}} \mathrm{t}=\frac{\|v\|}{\mathrm{p}(\|v\|)} \int_{0}^{1} \varphi(\mathrm{t}) \frac{v(\mathrm{t})}{\|v\|} \mathrm{p}(\|v\|) \mathrm{d}_{\mathrm{q}} \mathrm{t} \leqslant \frac{\|v\|}{\mathrm{p}(\|v\|)} \int_{0}^{1} \varphi(\mathrm{t}) \mathrm{p}(v(\mathrm{t})) \mathrm{d}_{\mathrm{q}} \mathrm{t} \leqslant \frac{\|v\|}{\mathrm{p}(\|v\|)} \mathcal{N}_{1} .
$$

Hence, $p(\|v\|) \leqslant \kappa_{1}^{-1} \mathcal{N}_{1}$. From $(\mathrm{H} 3), \lim _{z \rightarrow+\infty} p(z)=+\infty$, and thus there exists $\mathcal{N}_{2}>0$ such that $\|v\| \leqslant \mathcal{N}_{2}$. 
As a result, we can take $R>\max \left\{\kappa_{3}, \frac{c_{5} \kappa_{2}}{\kappa_{1}\left(d_{1} d_{2} \kappa_{1}^{2}-1\right)}, \mathcal{N}_{2}\right\}$ such that (3.1) holds true. Lemma 2.9 implies

$$
i\left(\mathcal{T}, B_{R} \cap(P \times P), P \times P\right)=0 .
$$

From (H4) we have

$$
\mathcal{T}_{1}(\mathrm{u}, v)(\mathrm{t})=\int_{0}^{1} \mathrm{G}(\mathrm{t}, \mathrm{qs}) \mathrm{F}_{1}(\mathrm{~s}, \mathrm{u}(\mathrm{s})-w(\mathrm{~s}), v(\mathrm{~s})-w(\mathrm{~s})) \mathrm{d}_{\mathrm{q}} \mathrm{s} \leqslant \int_{0}^{1} \varphi(\mathrm{s}) \mathcal{M} \mathcal{M}_{1} \mathrm{~d}_{\mathrm{q}} \mathrm{s}<\mathrm{k}_{3}=\|\mathrm{u}\| .
$$

This implies $\left\|\mathcal{T}_{1}(\mathfrak{u}, v)\right\|<\|u\|$. Similarly, $\left\|\mathcal{T}_{2}(u, v)\right\|<\|v\|$. Hence, $\|\mathcal{T}(u, v)\|<\|(u, v)\|$ for $u, v \in \partial B_{3} \cap P$. This indicates

$$
(u, v) \neq \lambda \mathcal{T}(u, v), \forall(u, v) \in \partial B_{3} \cap(P \times P), \lambda \in[0,1] .
$$

It follows from Lemma 2.10 that

$$
i\left(\mathcal{T}, B_{3} \cap(P \times P), P \times P\right)=1
$$

From (3.3) and (3.4) we have

$$
i\left(\mathcal{T},\left(B_{R} \backslash \bar{B}_{3}\right) \cap(P \times P), P \times P\right)=0-1=-1 .
$$

Therefore the operator $\mathcal{T}$ has at least one fixed point in $\left(B_{R} \backslash \bar{B}_{3}\right) \cap(P \times P)$, and then (1.1) has at least a positive solution. This completes the proof.

Theorem 3.2. Suppose that (H1), (H2), (H5), (H6) hold. Then (1.1) has at least one positive solution.

Proof. By (H5) we have

$$
\mathcal{T}_{1}(\mathrm{u}, v)\left(\mathrm{t}_{0}\right)=\int_{0}^{1} \mathrm{G}\left(\mathrm{t}_{0}, \mathrm{q} s\right) \mathrm{F}_{1}(\mathrm{~s}, \mathrm{u}(\mathrm{s})-w(\mathrm{~s}), v(\mathrm{~s})-w(\mathrm{~s})) \mathrm{d}_{\mathrm{q}} \mathrm{s} \geqslant \int_{\theta}^{1} \mathrm{t}_{0}^{\alpha-1} \varphi(\mathrm{s}) \mathcal{M}_{2} \mathrm{~d}_{\mathrm{q}} \mathrm{s} \geqslant \mathrm{k}_{3} .
$$

Hence, $\left\|\mathcal{T}_{1}(u, v)\right\| \geqslant \mathcal{T}_{1}(u, v)\left(t_{0}\right) \geqslant\|u\|$. Similarly, $\left\|\mathcal{T}_{2}(u, v)\right\| \geqslant \mathcal{T}_{2}(u, v)\left(t_{0}\right) \geqslant\|v\|$. This implies $\|\mathcal{T}(u, v)\| \geqslant$ $\|(u, v)\|$ for $u, v \in \partial B_{3} \cap P$. This yields

$$
(u, v) \neq \mathcal{T}(u, v)+\lambda(\psi, \psi), \quad \forall(u, v) \in \partial B_{3} \cap(P \times P), \lambda \geqslant 0,
$$

where $\psi \in \mathrm{P}_{0}$ is a given function. Lemma 2.9 implies

$$
i\left(\mathcal{T}, B_{3} \cap(P \times P), P \times P\right)=0 .
$$

On the other hand, we prove that there exists an adequately big positive number $R>\kappa_{3}$ such that

$$
(u, v) \neq \lambda \mathcal{T}(u, v), \quad \forall(u, v) \in \partial B_{R} \cap(P \times P), \lambda \in[0,1] .
$$

If not, there exist $(u, v) \in \partial B_{R} \cap(P \times P)$ and $\lambda \in[0,1]$ such that $(u, v)=\lambda \mathcal{T}(u, v)$, and then $u(t) \leqslant$ $\mathcal{T}_{1}(\mathrm{u}, v)(\mathrm{t}), v(\mathrm{t}) \leqslant \mathcal{T}_{2}(\mathrm{u}, v)(\mathrm{t})$ for $\mathrm{t} \in[0,1]$. By $(\mathrm{H} 6)$ we have

$$
\begin{aligned}
& u(t) \leqslant \int_{0}^{1} G(t, q s)\left[d_{3}(u(s)-w(s))+d_{4}(v(s)-w(s))+c_{1}^{\prime}\right] d_{q} s \leqslant \int_{0}^{1} G(t, q s)\left(d_{3} u(s)+d_{4} v(s)\right) d_{q} s+c_{6}, \\
& v(t) \leqslant \int_{0}^{1} G(t, q s)\left[d_{5}(u(s)-w(s))+d_{6}(v(s)-w(s))+c_{1}^{\prime}\right] d_{q} s \leqslant \int_{0}^{1} G(t, q s)\left(d_{5} u(s)+d_{6} v(s)\right) d_{q} s+c_{6} .
\end{aligned}
$$

Now multiplying by $\varphi(t)$ and integrating over $[0,1]$, by means of $(2.6)$ we get

$$
\begin{aligned}
& \int_{0}^{1} \varphi(t) u(t) d_{q} t \leqslant \kappa_{2} \int_{0}^{1} \varphi(t)\left(d_{3} u(t)+d_{4} v(t)\right) d_{q} t+c_{6} \kappa_{2}, \\
& \int_{0}^{1} \varphi(t) v(t) d_{q} t \leqslant \kappa_{2} \int_{0}^{1} \varphi(t)\left(d_{5} u(t)+d_{6} v(t)\right) d_{q} t+c_{6} \kappa_{2} .
\end{aligned}
$$


This implies

$$
\left[\begin{array}{cc}
1-\kappa_{2} d_{3} & -\kappa_{2} d_{4} \\
-\kappa_{2} d_{5} & 1-\kappa_{2} d_{6}
\end{array}\right]\left[\begin{array}{c}
\int_{0}^{1} \varphi(t) u(t) d_{q} t \\
\int_{0}^{1} \varphi(t) v(t) d_{q} t
\end{array}\right] \leqslant\left[\begin{array}{l}
c_{6} \kappa_{2} \\
c_{6} \kappa_{2}
\end{array}\right]
$$

Hence, we have

$$
\left[\begin{array}{c}
\int_{0}^{1} \varphi(t) u(t) d_{q} t \\
\int_{0}^{1} \varphi(t) v(t) d_{q} t
\end{array}\right] \leqslant \frac{1}{\Delta}\left[\begin{array}{cc}
1-\kappa_{2} d_{6} & \kappa_{2} d_{4} \\
\kappa_{2} d_{5} & 1-\kappa_{2} d_{3}
\end{array}\right]\left[\begin{array}{l}
c_{6} \kappa_{2} \\
c_{6} \kappa_{2}
\end{array}\right] .
$$

Therefore, there exist $\mathcal{N}_{3}>0, \mathcal{N}_{4}>0$ such that

$$
\left[\begin{array}{l}
\int_{0}^{1} \varphi(t) u(t) d_{q} t \\
\int_{0}^{1} \varphi(t) v(t) d_{q} t
\end{array}\right] \leqslant\left[\begin{array}{l}
\mathcal{N}_{3} \\
\mathcal{N}_{4}
\end{array}\right]
$$

Note that $u, v \in \mathrm{P}_{0}$, we have

$$
\|\mathrm{u}\| \leqslant \kappa_{1}^{-1} \mathcal{N}_{3},\|v\| \leqslant \kappa_{1}^{-1} \mathcal{N}_{4}
$$

As a result, we can take $R>\max \left\{\kappa_{3}, \kappa_{1}^{-1} \mathcal{N}_{3}, \kappa_{1}^{-1} \mathcal{N}_{4}\right\}$ such that (3.6) holds true. Lemma 2.10 implies

$$
i\left(\mathcal{T}, B_{R} \cap(P \times P), P \times P\right)=1 .
$$

From (3.5) and (3.7) we have

$$
i\left(\mathcal{T},\left(B_{R} \backslash \bar{B}_{3}\right) \cap(P \times P), P \times P\right)=1-0=1 .
$$

Therefore the operator $\mathcal{T}$ has at least one fixed point in $\left(B_{R} \backslash \bar{B}_{3}\right) \cap(P \times P)$, and thus (1.1) has at least a positive solution. This completes the proof.

\section{Acknowledgment}

This work was supported by the National Natural Science Foundation of China (Grant No. 11601048), Natural Science Foundation of Chongqing (Grant No. cstc2016jcyjA0181), the Science and Technology Research Program of Chongqing Municipal Education Commission (Grant No. KJ1703050, KJ1703043), and Natural Science Foundation of Chongqing Normal University (Grant No. 16XYY24, 15XLB011).

\section{References}

[1] R. P. Agarwal, Certain fractional q-integrals and q-derivatives, Proc. Cambridge Philos. Soc., 66 (1969), 365-370. 1, $2.2,2$

[2] Y. Cui, Y. Zou, An existence and uniqueness theorem for a second order nonlinear system with coupled integral boundary value conditions, Appl. Math. Comput., 256 (2015), 438-444.

[3] R. Dahal, D. Duncan, C. S. Goodrich, Systems of semipositone discrete fractional boundary value problems, J. Difference Equ. Appl., 20 (2014), 473-491. 1

[4] R. A. C. Ferreira, Nontrivial solutions for fractional q-difference boundary-value problems, Electron. J. Qual. Theory Differ. Equ., 2010 (2010), 10 pages. 2.1, 2

[5] R. A. C. Ferreira, Positive solutions for a class of boundary value problems with fractional q-differences, Comput. Math. Appl., 61 (2011), 367-373. 1, 2.7, 2

[6] D. Guo, V. Lakshmikantham, Nonlinear Problems in Abstract Cones, Academic Press, Boston, (1988). 2.9, 2.10

[7] J. Henderson, R. Luca, Existence of positive solutions for a system of semipositone fractional boundary value problems, Electron. J. Qual. Theory Differ. Equ., 2016 (2016), 28 pages. 1

[8] J. Jiang, L. Liu, Y. Wu, Positive solutions to singular fractional differential system with coupled boundary conditions, Commun. Nonlinear Sci. Numer. Simulat., 18 (2013), 3061-3074.

[9] V. Kac, P. Cheung, Quantum Calculus, Springer, New York, (2002). 2

[10] A. Kilbas, H. Srivastava, J. Trujillo, Theory and Applications of Fractional Differential Equations, Elsevier Science B.V., Amsterdam, (2006).

[11] B. Li, S. Sun, Z. Han, Positive solutions for singular fractional differential equations with three-point boundary conditions, J. Appl. Math. Comput., 52 (2016), 477-488. 
[12] Y. Li, Z. Wei, Positive solutions for a coupled systems of mixed higher-order nonlinear singular fractional differential equations, Fixed Point Theory, 15 (2014), 167-178.

[13] Y. Liu, New existence results for positive solutions of boundary value problems for coupled systems of multi-term fractional differential equations, Hacet. J. Math. Stat., 45 (2016), 391-416.

[14] R. Luca, A. Tudorache, Positive solutions to a system of semipositone fractional boundary value problems, Adv. Difference Equ., 2014 (2014), 11 pages. 1

[15] I. Podlubny, Fractional Differential Equations, Academic Press, San Diego, (1999).

[16] P. M. Rajković, S. D. Marinković, M. S. Stanković, Fractional integrals and derivatives in q-calculus, Appl. Anal. Discrete Math., 1 (2007), 311-323. 2.3, 2

[17] S. Samko, A. Kilbas, O. Marichev, Fractional Integrals and Derivatives: Theory and Applications, Gordon and Breach, USA, (1993).

[18] X. Su, Boundary value problem for a coupled systemof nonlinear fractional differential equations, Appl. Math. Lett., 22 (2009), 64-69.

[19] Y. Wang, L. Liu, X. Zhang, Y. Wu, Positive solutions of an abstract fractional semipositone differential system model for bioprocesses of HIV infection, Appl. Math. Comput., 258 (2015), 312-324. 1

[20] W. Yang, Positive solutions for nonlinear semipositone fractional q-difference system with coupled integral boundary conditions, Appl. Math. Comput., 244 (2014), 702-725.

[21] C. Yuan, Two positive solutions for $(n-1,1)$-type semipositone integral boundary value problems for coupled systems of nonlinear fractional differential equations, Commun. Nonlinear Sci. Numer. Simul., 17 (2012), 930-942. 1

[22] X. Zhang, L. Liu, Y. Wu, The uniqueness of positive solution for a singular fractional differential system involving derivatives, Commun. Nonlinear Sci. Numer. Simul., 18 (2013), 1400-1409.

[23] X. Zhang, L. Liu, Y. Wu, The uniqueness of positive solution for a fractional order model of turbulent flow in a porous medium, Appl. Math. Lett., 37 (2014), 26-33.

[24] X. Zhang, L. Liu, Y. Wu, B. Wiwatanapataphee, The spectral analysis for a singular fractional differential equation with a signed measure, Appl. Math. Comput., 257 (2015), 252-263.

[25] K. Zhang, J. Xu, D. O'Regan, Positive solutions for a coupled system of nonlinear fractional differential equations, Math. Meth. Appl. Sci., 38 (2015), 1662-1672. 1, 1 